\title{
EX VITRO ROOTING, ACCLIMATIZATION AND GENETIC STABILITY OF LONICERA CAERULEA VAR. KAMTSCHATICA
}

\author{
Agnieszka WOJTANIA*, Monika MARKIEWICZ, Justyna GÓRAJ-KONIARSKA \\ Research Institute of Horticulture, Konstytucji 3 Maja 1/3 St., 96-100 Skierniewice, Poland
}

Received: May 2020; Accepted: September 2020

\begin{abstract}
Ex vitro rooting and acclimatization of two cultivars 'Wojtek' and 'Zojka' of blue honeysuckle (Lonicera caerulea var. kamtschatica Sevast.) were studied. To the ex vitro conditions were transferred rooted and unrooted shoots. The post-effect of auxin type and concentration as well as microcutting and soil substrate types were tested. The genetic stability of the plantlets in relation to the mother plants by using amplified fragment length polymorphism (AFLP) and inter simple sequence repeat (ISSR) markers has been also determined. It has been found that in vitro rooted cuttings of both cultivars showed a higher survival rate (max. 88\%) and better growth and development when they were rooted on a medium containing a low auxin level $\left(1.0 \mathrm{mg} \cdot \mathrm{dm}^{-3}\right)$. The results of the second experiment showed successful ex vitro rooting of blue honeysuckle shoots without auxin treatment. Higher ex vitro rooting and survival rate in the greenhouse have been observed for 'Wojtek' (max. 96\%) than 'Zojka' (max. 88\%). Better growth and development of shoots and roots were observed on peat alone or a mixture of peat and perlite as compared to a mixture of peat and sand. The micropropagated plantlets appeared similar to mother plants. Molecular analysis confirmed a high level of genetic stability of blue honeysuckle after 2 years of in vitro propagation. However, among the cultivars studied, 'Wojtek' showed slightly higher genetic stability than 'Zojka' (99.5\% and 97.7\%, respectively). For 'Zojka' plants, the degree of variation was comparable for AFLP and ISSR markers. For 'Wojtek', no polymorphism was detected using the ISSR analysis in contrast to the AFLP analysis.
\end{abstract}

Keywords: AFLP, blue honeysuckle, ISSR, micropropagation

\section{INTRODUCTION}

Blue honeysuckle (Lonicera caerulea $\mathrm{L}$. var. kamtschatica Sevast.) belongs to the family Caprifoliaceae, genus Lonicera, which includes about 200 species (Poyarkova 2000). It has been growing wild in Siberia for millennia and was treasured for its nutritional value. Nowadays, it is cultivated across Japan, China, Russia, Central and Eastern Europe, especially Poland, the Czech Republic, Slovenia, Slovakia, and North America, Canada and the USA. Blue honeysuckle, also called "sweet berry honeysuckle", "haskap berry" or "edible honeysuckle", is a shrub that grows up to $2 \mathrm{~m}$. It is a long-lived plant and can bear fruit up to 30 years. In addition, it is resistant to diseases, pests and frost. The fruits are rich in bioactive compounds, polyphenols, especially anthocyanins, and exhibit anti-inflammatory and bacteriostatic properties (Plekhanova 2000; Svarcova et al. 2007; Jurikova et al. 2011).
The fruits can be sold as fresh or frozen products or used for juices, jams or even wine and gin products. An improved understanding of the role of dietary value of fruit in maintaining human health caused a noticeable increase of interest in the cultivation of this plant. In December 13, 2018 under regulation no. 2015/2283 of the European Parliament, fruits of L. caerulea $\mathrm{L}$. were entered on the list of novel foods. This gave the opportunity to legally market blue honeysuckle berries in the European Union.

Honeysuckle species are propagated traditionally by semi-hardwood and softwood cuttings. This method, though generally successful, largely depends on the individual genotype, age of the stock plant and vegetation period (Hui et al. 2012). Tissue culture offers an alternative method of plant propagation, which is independent of the vegetative season. High multiplication rates and good health status of micropropagated plants are the additional features of this method. 
Most of the studies on in vitro culture of blue honeysuckle involved micropropagation by axillary shoots in the presence of 6-benzylaminopurine (BAP) (Karhu 1997a; Sedlák \& Paprštein 2007; Dziedzic 2008; Krupa-Małkiewicz \& Ochmian 2014) or the mixture of 2-isopentyladenine (2iP) and 3-hydroxybenzyladenine (meta-topolin) (Gabryszewska et al. 2016). In vitro rooting of shoots has been successfully achieved by using auxin treatment (Karhu 1997b; Dziedzic 2008; Krupa-Małkiewicz et al. 2017). On an auxin-free medium, the rooting rate was low, for example, $4-8 \%$ for 'Wojtek' and $40 \%$ for 'Zojka', and a weak root system has been observed (Krupa-Małkiewicz et al. 2017, Wojtania et al. 2018). There is so far no information on ex vitro rooting and acclimatization of blue honeysuckle microshoots. The success of micropropagation is clearly dependent on acclimatization of plantlets to the ex vitro condition and maintaining the genetic stability of propagules.

Under long-term in vitro propagation, various factors such as genotype, explant type and origin, media composition, plant growth regulators and cultural environment may induce the variation in plant material (Podwyszyńska 2006; Olhoft \& Phillips 1999; Bednarek \& Orłowska 2020). Various types of phenotypic and genetic changes have been observed including polyploidy, aneuploidy and other types of mutations such as, e.g., point mutations or insertions of transposons but also changes resulting from, for example, DNA methylation or histone modifications. These modifications may influence gene transcription. Epigenetic changes are often temporary, and plants may revert to the normal phenotype relatively easily (Jain 2001; Smulders \& de Klerk 2011). Molecular markers become the most desirable tool for establishing genetic uniformity of in vitro derived plantlets of different plant species, including berry plants such as lowbush blueberry, strawberry and blackberry (Debnath 2011, 2013; Borsai et al. 2020). To date, there is no genetic stability assessment system for micropropagated plants of blue honeysuckle.

The aim of this study was to develop an effective method of ex vitro rooting and acclimatization of microcutting of two L. caerulea var. kamtschatica cultivars 'Wojtek' and 'Zojka'. The genetic stability of plantlets in relation to the mother plants by using amplified fragment length polymorphism (AFLP) and inter simple sequence repeat (ISSR) markers has been also determined.

\section{MATERIALS AND METHODS}

\section{Plant material}

Axillary shoot cultures of two L. caerulea var. kamtschatica cultivars 'Wojtek' and 'Zojka' were initiated from 2-year-old nursery plants. Shoots were established and continuously multiplied for 2 years on the Murashige and Skoog (1962) medium (MS) modified with a supplementation $85.5 \mathrm{mg} \cdot \mathrm{dm}^{-3} \mathrm{NaH}_{2} \mathrm{PO}_{4} \cdot \mathrm{H}_{2} \mathrm{O}, 100 \mathrm{mg} \cdot \mathrm{dm}^{-3}$ myoinositol, nicotinic acid, pyridoxine and thiamine $\left(1.0 \mathrm{mg} \cdot \mathrm{dm}^{-3}\right.$ each), $15 \mathrm{mg} \cdot \mathrm{dm}^{-3} 2 \mathrm{iP}, 1.0 \mathrm{mg} \cdot \mathrm{dm}^{-3}$ meta-topolin, $30 \mathrm{~g} \cdot \mathrm{dm}^{-3}$ sucrose and solidified with a mixture of gelling agents, $3 \mathrm{~g} \cdot \mathrm{dm}^{-3}$ agar (Biocorp, Poland) and $1.2 \mathrm{~g} \cdot \mathrm{dm}^{-3}$ Gelrite (Duchefa, Netherlands) (Gabryszewska et al. 2016). The $\mathrm{pH}$ of the medium was adjusted to 5.6 before autoclaving. Axillary shoot cultures were subcultured on the fresh medium every 6-8 weeks.

\section{Acclimatization of in vitro rooted shoots (Exper- iment 1)}

The shoots were rooted in vitro on the MS medium containing indole-3-butyric acid (IBA) or 1-naphthaleneacetic acid (IAA) at different concentrations (0.0; 1.0; 2.5; and $4.0 \mathrm{mg} \cdot \mathrm{dm}^{-3}$ ) (Wojtania et al. 2018). The aim of this experiment was to determine the posteffect of auxin type and concentrations on ex vitro acclimatization. The in vitro rooted microcuttings were planted at the end of March in multi-cell plug trays of $30 \mathrm{~mm}$ diameter, with peat substrate, in plastic mini-greenhouses (Garland Products, UK) with two adjustable "dial" ventilators to control the humidity and optimize growing conditions. The plants were maintained for 4 weeks in a growth room under fluorescent lamps $\left(50 \mu \mathrm{mol} \cdot \mathrm{m}^{-2} \cdot \mathrm{s}^{-1}\right)$ at $23 \pm 2{ }^{\circ} \mathrm{C}$. Starting with day 7 after the transfer to ex vitro conditions, the humidity was stepwise reduced by opening the ventilators and the plantlets were fertilized with Peters Professional Plant Starter $\left(0.25 \mathrm{~g} \cdot \mathrm{dm}^{-3}\right)$. After 6 weeks, the following data were collected: survival rate, fresh mass and length of cuttings as well as fresh mass, the number and length of roots.

Ex vitro direct rooting and acclimatization (Experiment 2)

The aim of this experiment was to determine ex vitro rooting and acclimatization ability in the greenhouse of axillary shoots derived from a 6-week-old in vitro cultures incubated on a multiplication medium. 
For ex vitro direct rooting, two types of explants were used: single microshoots about $1.0 \mathrm{~cm}$ long and clumps of 4-5 shoots. The experiment started in mid-June. Both explant types were planted in multi cell plug trays of $30 \mathrm{~mm}$ diameter with three different soil substrates peat, peat + sand $(2: 1 \mathrm{v} / \mathrm{v})$, peat + perlite $(2: 1)-$ in plastic mini-greenhouses maintained in the greenhouse. Cuttings were maintained under natural photoperiod and irradiance with day/night of $24 \pm 5^{\circ} \mathrm{C} / 18 \pm 5^{\circ} \mathrm{C}$. The acclimatization procedure was the same as in $\mathrm{Ex}$ periment 1 . After 6 weeks of growing ex vitro in the greenhouse, the following data were collected: survival and rooting rate, fresh mass and length of cutting as well as fresh mass, the number and length of roots.

\section{Genetic stability assessment}

The genetic stability of acclimatized plantlets of two L. caerulea var. kamtschatica cultivars ('Wojtek' and 'Zojka') was evaluated in relation to the mother plants (standards) by using ISSR and AFLP analyses.

DNA was extracted from fresh leaves collected from 15 randomly selected acclimatized microplants of each cultivar. Genomic DNA was extracted using the Gene MATRIX Plant \& Fungi DNA Purification Kit (EURx) in two replicates for each sample tested. The concentration and purity of the DNA were determined using an Epoch spectrophotometer (BioTek). Four ISSR and four AFLP primer pairs were finally used in the study after an initial screening of 23 ISSR and 23 AFLP primer pairs for the production of a high number of distinct and countable bands (Tab. 1). For ISSR analysis, the PCR reaction was carried out in $20 \mu \mathrm{L}$ reaction volume containing 20 ng DNA, $1 \times$ DreamTaq $^{\mathrm{TM}}$ Green Buffer (Thermo Fisher Scientific), $0.65 \mu \mathrm{L}$ dNTPs (10 mM) (Promega), $0.45 \mu \mathrm{L}$ ISSR primer $(10 \mu \mathrm{M})$ and $0.5 \mathrm{U}$ DreamTaq ${ }^{\mathrm{TM}}$ Green Polymerase (Thermo Fisher Scientific). Amplification was carried out in the T100 Thermal Cycler (BioRad) programmed for 45 ISSR cycles $\left(30 \mathrm{~s}\right.$ at $94{ }^{\circ} \mathrm{C}, 40 \mathrm{~s}$ at $55^{\circ} \mathrm{C}, 90 \mathrm{~s}$ at $72{ }^{\circ} \mathrm{C}$ ). All reactions were repeated twice. The PCR products obtained were separated on $1.5 \%$ agarose gel through electrophoresis and photographed using Syngen Biotech camera. The size of the bands was assessed against size standards GeneRuler $^{\mathrm{TM}} 100$ bp DNA Ladder Plus (Thermo Fisher Scientific). For AFLP analysis, genomic DNA (50 ng) was digested with $M s e \mathrm{I}$ and PstI endonucleases and ligated with appropriate adapters (Vos et al. 1995).
Preamplification of obtained fragments was carried out using primers complementary to the adapter's sequence. The preselection PCR reaction mixture contains $20 \mu \mathrm{L}$ DNA, $1 \times$ Taq Polymerase Reaction Buffer (Sigma-Aldrich), $0.8 \mu \mathrm{L}$ dNTPs $(10 \mathrm{mM})$ (Promega), $1.2 \mu \mathrm{L}$ of each primer $(10 \mu \mathrm{M})$ and $0.75 \mathrm{U}$ Taq DNA Polymerase (Sigma-Aldrich). Amplifications were carried out in the thermal cycler programmed for 30 cycles $\left(30 \mathrm{~s}\right.$ at $94{ }^{\circ} \mathrm{C}, 30 \mathrm{~s}$ at $60^{\circ} \mathrm{C}, 60 \mathrm{~s}$ at $72{ }^{\circ} \mathrm{C}$ ). The selective PCR with primer pairs was obtained by extending the primers used in preamplification with two additional nucleotides at the 3 ' end (Tab. 1). PCR was carried out in the reaction mixture containing $3 \mu \mathrm{L}$ DNA $1: 30(\mathrm{v}: \mathrm{v}), 1 \times$ Taq Polymerase Reaction Buffer (Sigma-Aldrich), $0.4 \mu \mathrm{L}$ dNTPs $(10 \mathrm{mM})$ (Promega), $0.5 \mu \mathrm{L}$ PstI-NN primer $(10 \mu \mathrm{M}), 0.65 \mu \mathrm{L}$ MseI-NN primer $(10 \mu \mathrm{M})$ and $0.75 \mathrm{U}$ Taq DNA Polymerase (Sigma-Aldrich). Amplification was carried out in the thermal cycler programmed for 13 cycles $\left(30 \mathrm{~s}\right.$ at $94{ }^{\circ} \mathrm{C}, 30 \mathrm{~s}$ at $65-56{ }^{\circ} \mathrm{C}$ with annealing temperature decreased by $0.7^{\circ} \mathrm{C}$ in each cycle, $60 \mathrm{~s}$ at $72{ }^{\circ} \mathrm{C}$ ), followed by 26 cycles $\left(30 \mathrm{~s}\right.$ at $94{ }^{\circ} \mathrm{C}, 30 \mathrm{~s}$ at $56{ }^{\circ} \mathrm{C}, 60 \mathrm{~s}$ at $72{ }^{\circ} \mathrm{C}$ ). Products of selective PCR were separated on $6 \%$ denaturing polyacrylamide gel through electrophoresis on Dual Dedicated Height Nucleic Acid Sequencer (C.B.S. Scientific). The separated AFLP products were stained in a silver nitrate solution and the gel was dried, described and photographed. The size of the bands was assessed against size standards 10 bp DNA Ladder (Invitrogen) and $50 \mathrm{bp}$ DNA Ladder (Invitrogen). Bands generated in ISSR and AFLP analysis were scored manually as present (1) or absent (0) from the photographs. Only bright and reproducible products were scored. During the analysis of electrophoregrams, the number of ISSR and AFLP products, their size and diversity between mother plants and plants obtained from micropropagation were evaluated.

\section{Statistical analysis}

In all experiments on the rooting and acclimatization, 25 microcuttings for each treatment were taken. The experiments were carried out twice. The results were statistically analyzed with the use of the STATISTICA program (13.1 PL 2012, Statsoft, Poland). An analysis of variance was performed with ANOVA, R.A. Fischer for a two-factor system. Means were compared using Duncan's test at $\mathrm{p}=0.05$. 
Table 1. Primers, polymorphism between tested cultivars and amplification products of ISSR-PCR and AFLPPCR used for evaluation of genetic identity of Lonicera caerulea 'Wojtek' and 'Zojka' plants

\begin{tabular}{|c|c|c|c|c|c|c|c|c|}
\hline No. & Primer & $\begin{array}{l}\text { Sequence } \\
\left(5^{\prime}-33^{\prime}\right)\end{array}$ & $\begin{array}{c}\text { Total } \\
\text { number } \\
\text { of bands }\end{array}$ & $\begin{array}{c}\text { Polymorphic } \\
\text { bands } \\
(\%)\end{array}$ & Primer & $\begin{array}{l}\text { Sequence } \\
\left(5^{\prime}-3^{\prime}\right)\end{array}$ & $\begin{array}{c}\text { Total } \\
\text { number } \\
\text { of bands }\end{array}$ & $\begin{array}{c}\text { Polymorphic } \\
\text { bands } \\
(\%)\end{array}$ \\
\hline & \multicolumn{4}{|c|}{ ISSR } & \multicolumn{4}{|c|}{ AFLP } \\
\hline 1 & UBC 806 & $(\mathrm{TA})_{8} \mathrm{G}$ & - & - & Pst-TT/Mse-CC* & $\begin{array}{l}\text { GACTGCGTACATGCAGTT } \\
\text { GATGAGTCCTGAGTAACC }\end{array}$ & 30 & 13.3 \\
\hline 2 & UBC $810^{*}$ & $(\mathrm{GA})_{8} \mathrm{~T}$ & 6 & 16.7 & Pst-GC/Mse-GC & $\begin{array}{l}\text { GACTGCGTACATGCAGGC } \\
\text { GATGAGTCCTGAGTAAGC }\end{array}$ & 21 & 0 \\
\hline 3 & UBC 813 & $(\mathrm{CT})_{8} \mathrm{~T}$ & - & - & Pst-GC/Mse-TA* & $\begin{array}{l}\text { GACTGCGTACATGCAGGC } \\
\text { GATGAGTCCTGAGTAATA }\end{array}$ & 18 & 11.1 \\
\hline 4 & UBC 821 & $(\mathrm{GT})_{8} \mathrm{~T}$ & - & - & Pst-TA/Mse-TA & $\begin{array}{l}\text { GACTGCGTACATGCAGTA } \\
\text { GATGAGTCCTGAGTAATA }\end{array}$ & 14 & 0 \\
\hline 5 & UBC 822 & $(\mathrm{TC})_{8} \mathrm{~A}$ & 9 & 0 & Pst-TA/Mse-GC & $\begin{array}{l}\text { GACTGCGTACATGCAGTA } \\
\text { GATGAGTCCTGAGTAAGC }\end{array}$ & 42 & 0 \\
\hline 6 & UBC 823 & $(\mathrm{TC})_{8} \mathrm{C}$ & 5 & 0 & Pst-AG/Mse-AG & $\begin{array}{l}\text { GACTGCGTACATGCAGAG } \\
\text { GATGAGTCCTGAGTAAAG }\end{array}$ & 54 & 0 \\
\hline 7 & UBC $825^{*}$ & $(\mathrm{AC})_{8} \mathrm{~T}$ & 7 & 28.6 & Pst-AG/Mse-CG & $\begin{array}{l}\text { GACTGCGTACATGCAGAG } \\
\text { GATGAGTCCTGAGTAACG }\end{array}$ & 36 & 0 \\
\hline 8 & UBC $827 *$ & $(\mathrm{AC})_{8} \mathrm{G}$ & 12 & 8.3 & Pst-CG/Mse-AG* & $\begin{array}{l}\text { GACTGCGTACATGCAGCG } \\
\text { GATGAGTCCTGAGTAAAG }\end{array}$ & 42 & 4.8 \\
\hline 9 & UBC 828 & $(\mathrm{TG})_{8} \mathrm{~A}$ & 2 & 0 & Pst-TC/Mse-TC & $\begin{array}{l}\text { GACTGCGTACATGCAGTC } \\
\text { GATGAGTCCTGAGTAATC }\end{array}$ & 13 & 0 \\
\hline 10 & UBC 830 & $(\mathrm{TG})_{8} \mathrm{G}$ & 1 & 0 & Pst-TC/Mse-AT & $\begin{array}{l}\text { GACTGCGTACATGCAGTC } \\
\text { GATGAGTCCTGAGTAAAT }\end{array}$ & 20 & 0 \\
\hline 11 & UBC 834 & $(\mathrm{AG})_{8} \mathrm{CT}$ & 3 & 0 & Pst-AT/Mse-AT & $\begin{array}{l}\text { GACTGCGTACATGCAGAT } \\
\text { GATGAGTCCTGAGTAAAT }\end{array}$ & 50 & 0 \\
\hline 12 & UBC 840 & $(\mathrm{GA})_{8} \mathrm{CT}$ & 2 & 0 & Pst-AT/Mse-TC & $\begin{array}{l}\text { GACTGCGTACATGCAGAT } \\
\text { GATGAGTCCTGAGTAATC }\end{array}$ & 25 & 0 \\
\hline 13 & UBC 843 & $(\mathrm{CT})_{8} \mathrm{GA}$ & 1 & 0 & Pst-AA/Mse-AA & $\begin{array}{l}\text { GACTGCGTACATGCAGAA } \\
\text { GATGAGTCCTGAGTAAAA }\end{array}$ & 13 & 0 \\
\hline 14 & UBC 846 & $(\mathrm{CA})_{8} \mathrm{GT}$ & 3 & 0 & Pst-AA/Mse-AC & $\begin{array}{l}\text { GACTGCGTACATGCAGAA } \\
\text { GATGAGTCCTGAGTAAAC }\end{array}$ & 37 & 0 \\
\hline 15 & UBC 853 & (TC) ${ }_{8} \mathrm{AT}$ & 6 & 0 & Pst-AC/Mse-AC & $\begin{array}{l}\text { GACTGCGTACATGCAGAC } \\
\text { GATGAGTCCTGAGTAAAC }\end{array}$ & 12 & 0 \\
\hline 16 & UBC 848 & $(\mathrm{CA})_{8} \mathrm{GG}$ & - & - & Pst-AC/Mse-AA & $\begin{array}{l}\text { GACTGCGTACATGCAGAC } \\
\text { GATGAGTCCTGAGTAAAA }\end{array}$ & 8 & 0 \\
\hline 17 & UBC 849 & $(\mathrm{GT})_{8} \mathrm{CA}$ & - & - & Pst-GG/Mse-GG & $\begin{array}{l}\text { GACTGCGTACATGCAGGG } \\
\text { GATGAGTCCTGAGTAAGG }\end{array}$ & - & - \\
\hline 18 & UBC 855 & $(\mathrm{AC})_{8} \mathrm{CT}$ & 4 & 0 & Pst-GG/Mse-GA & $\begin{array}{l}\text { GACTGCGTACATGCAGGG } \\
\text { GATGAGTCCTGAGTAAGA }\end{array}$ & - & - \\
\hline 19 & UBC 858 & $(\mathrm{TG})_{8} \mathrm{AT}$ & - & - & Pst-GA/Mse-GA & $\begin{array}{l}\text { GACTGCGTACATGCAGGA } \\
\text { GATGAGTCCTGAGTAAGA }\end{array}$ & 19 & 0 \\
\hline 20 & UBC $865^{*}$ & $(\mathrm{CCG})_{6}$ & 4 & 25.0 & Pst-GA/Mse-GC & $\begin{array}{l}\text { GACTGCGTACATGCAGGA } \\
\text { GATGAGTCCTGAGTAAGC }\end{array}$ & 9 & 0 \\
\hline 21 & UBC 867 & $(\mathrm{GGC})_{6}$ & - & - & Pst-CC/Mse-CC* & $\begin{array}{l}\text { GACTGCGTACATGCAGCC } \\
\text { GATGAGTCCTGAGTAACC }\end{array}$ & 20 & 30.0 \\
\hline 22 & UBC 875 & $(\mathrm{CTAG})_{4}$ & - & - & Pst-CC/Mse-GG & $\begin{array}{l}\text { GACTGCGTACATGCAGCC } \\
\text { GATGAGTCCTGAGTAAGG }\end{array}$ & 43 & 0 \\
\hline 23 & UBC 881 & $(\mathrm{GGGT})_{3} \mathrm{G}$ & - & - & Pst-TT/Mse-CC & $\begin{array}{l}\text { GACTGCGTACATGCAGTT } \\
\text { GATGAGTCCTGAGTAACC }\end{array}$ & 48 & 0 \\
\hline
\end{tabular}

* primers selected for analysis of genetic stability of micropropagated plants; "-“ no visible and scorable bands 


\section{RESULTS}

\section{Acclimatization of in vitro rooted shoots}

For both cultivars, the highest survival rate (max. 88\%) was observed for microcuttings rooted on the medium containing the lowest auxin level $\left(1.0 \mathrm{mg} \cdot \mathrm{dm}^{-3}\right)$. However, the differences between auxin type and concentration on survival rate in ex vitro conditions were not significant. It has been also found that the microcuttings rooted on low auxin medium after transfer to ex vitro conditions showed higher fresh mass and length of shoots as well as all root parameters (fresh mass, number and length) as compared to those rooted on the medium with high auxin (Tab. 2). During acclimatization, wilting and shoot tip necrosis were also observed, and these were most frequent among shoots rooted in vitro in the presence of high auxin concentration (data not shown).

\section{$E x$ vitro rooting and acclimatization}

The results showed successful ex vitro rooting without auxin in the greenhouse of both blue honeysuckle cultivars (Tab. 3). The ability to form roots depended on genotype. Higher ex vitro rooting and survival rate in the greenhouse were observed for 'Wojtek' (max. 96\%) than 'Zojka' (max. 88\%). There were no significant differences in rooting/survival rate of 'Zojka' either between microcutting types or soil substrates. However, the plantlets of 'Wojtek' showed a higher rooting/survival rate when using individual microcuttings than shoot clumps (Fig. 1). Shoot clumps had a higher tendency toward the wilting and the shoot tip necrosis, leading to the death of some plantlets. The plants of both cultivars that were acclimatized in peat alone or in a mixture of peat and perlite exhibited higher mass of shoots and roots as well as length of shoots as compared to those grown in a mixture of peat and sand (Tab. 3). The micropropagated plantlets appeared similar to mother plants. Genetic stability of in vitro propagated plants

The results showed that the ISSR primers generated a total of 40 and 36 amplification products ranging in size from 300 to $1400 \mathrm{bp}$ for 'Wojtek' and 'Zojka' plants, respectively (Tab. 4). Among these bands, $0.0 \%$ and $2.77 \%$ were polymorphic for 'Wojtek' and 'Zojka' cultivars in relation to the mother plants. The AFLP primer pairs generated a total of 102 and 105 amplification products ranging in size from 50 to $900 \mathrm{bp}$ for 'Wojtek' and 'Zojka' plants, respectively (Tab. 4). Among these bands, $0.98 \%$ and $1.90 \%$ were polymorphic for both 'Wojtek' and 'Zojka', compared to the mother plants.

Table 2. The post-effect of auxin type (IAA and IBA) and concentration $\left(0.0 ; 1.0 ; 2.5 ; 4.0 \mathrm{mg} \cdot \mathrm{dm}^{-3}\right)$ on ex vitro acclimatization of Lonicera caerulea 'Zojka' and 'Wojtek' in the growth chamber

\begin{tabular}{|c|c|c|c|c|c|c|c|c|}
\hline Genotype & $\begin{array}{r} \\
\text { type/cc } \\
(\mathrm{m}\end{array}$ & $\begin{array}{l}\text { in } \\
\text { ntration } \\
\mathrm{m}^{-3} \text { ) }\end{array}$ & $\begin{array}{c}\text { Survived } \\
\text { micro- } \\
\text { cutting } \\
(\%)\end{array}$ & $\begin{array}{c}\text { Fresh mass } \\
\text { of shoots } \\
\quad(\mathrm{mg})\end{array}$ & $\begin{array}{l}\text { Shoot length } \\
(\mathrm{mm})\end{array}$ & $\begin{array}{c}\text { Fresh root } \\
\text { mass } \\
(\mathrm{mg})\end{array}$ & $\begin{array}{c}\text { Root } \\
\text { number/cutting }\end{array}$ & $\begin{array}{l}\text { Root length } \\
\quad(\mathrm{mm})\end{array}$ \\
\hline \multirow{7}{*}{ 'Zojka' } & \multirow{4}{*}{ IBA } & 0.0 & $84 \mathrm{a}$ & $184 \pm 46.9 b c$ & $115 \pm 11.0 \mathrm{bc}$ & $52.6 \pm 20.7 \mathrm{c}$ & $2.5 \pm 0.9 \mathrm{c}$ & $38.5 \pm 9.8 \mathrm{ab}$ \\
\hline & & 1.0 & $88 \mathrm{a}$ & $247 \pm 69.7 \mathrm{a}$ & $154 \pm 21.7 \mathrm{a}$ & $72.2 \pm 35.0 \mathrm{~b}$ & $3.7 \pm 1.0 \mathrm{ab}$ & $37.1 \pm 10.7 \mathrm{ab}$ \\
\hline & & 2.5 & $80 a$ & $174 \pm 68.8 b c$ & $126 \pm 11.3 b$ & $63.6 \pm 23.4 b c$ & $3.8 \pm 0.7 \mathrm{a}$ & $32.4 \pm 15.8 b c$ \\
\hline & & 4.0 & $76 a$ & $179 \pm 63.5 b c$ & $116 \pm 17.4 b c$ & $54.8 \pm 11.9 b c$ & $3.7 \pm 1.0 \mathrm{ab}$ & $28.7 \pm 7.3 \mathrm{c}$ \\
\hline & \multirow{3}{*}{ IAA } & 1.0 & $80 \mathrm{a}$ & $219 \pm 77.4 \mathrm{ab}$ & $111 \pm 16.3 \mathrm{c}$ & $89.0 \pm 36.7 \mathrm{a}$ & $3.5 \pm 1.1 \mathrm{ab}$ & $42.0 \pm 14.0 \mathrm{a}$ \\
\hline & & 2.5 & $76 \mathrm{a}$ & $160 \pm 67.9 \mathrm{c}$ & $117 \pm 15.0 \mathrm{bc}$ & $48.4 \pm 14.9 \mathrm{c}$ & $3.3 \pm 0.9 \mathrm{ab}$ & $36.2 \pm 11.4 \mathrm{a}-\mathrm{c}$ \\
\hline & & 4.0 & $72 \mathrm{a}$ & $142 \pm 67.1 \mathrm{c}$ & $114 \pm 22.6 b c$ & $52.6 \pm 28.7 \mathrm{c}$ & $3.1 \pm 0.9 \mathrm{bc}$ & $33.1 \pm 7.1 b c$ \\
\hline \multirow{7}{*}{ 'Wojtek' } & \multirow{4}{*}{ IBA } & 0.0 & $80 \mathrm{a}$ & $218 \pm 85.1 b$ & $126 \pm 32.4 b$ & $42.7 \pm 20.4 \mathrm{~d}$ & $2.5 \pm 0.7 \mathrm{~d}$ & $51.3 \pm 8.5 b$ \\
\hline & & 1.0 & $88 \mathrm{a}$ & $349 \pm 95.4 \mathrm{a}$ & $181 \pm 23.1 \mathrm{a}$ & $120.1 \pm 43.5 \mathrm{a}$ & $3.7 \pm 1.1 \mathrm{ab}$ & $44.3 \pm 7.8 b c$ \\
\hline & & 2.5 & $84 \mathrm{a}$ & $198 \pm 73.8 b$ & $135 \pm 29.7 b$ & $75.6 \pm 22.4 b c$ & $3.6 \pm 0.9 \mathrm{ab}$ & $37.1 \pm 8.8 \mathrm{~cd}$ \\
\hline & & 4.0 & $68 \mathrm{a}$ & $160 \pm 81.5 b$ & $124 \pm 38.5 b$ & $74.8 \pm 25.7 b c$ & $3.2 \pm 0.7 \mathrm{~b}-\mathrm{d}$ & $33.3 \pm 8.5 \mathrm{~d}$ \\
\hline & \multirow{3}{*}{ IAA } & 1.0 & $84 \mathrm{a}$ & $339 \pm 50.3 \mathrm{a}$ & $146 \pm 32.4 \mathrm{~b}$ & $86.3 \pm 34.7 b$ & $4.1 \pm 1.2 \mathrm{a}$ & $59.0 \pm 18.9 \mathrm{a}$ \\
\hline & & 2.5 & $76 a$ & $210 \pm 61.1 b$ & $145 \pm 17.2 b$ & $70.8 \pm 26.4 b c$ & $3.4 \pm 1.1 \mathrm{bc}$ & $30.5 \pm 6.8 \mathrm{~d}$ \\
\hline & & 4.0 & $76 a$ & $216 \pm 58.4 \mathrm{~b}$ & $140 \pm 39.0 b$ & $58.5 \pm 21.8 \mathrm{~cd}$ & $2.9 \pm 1.0 \mathrm{~cd}$ & $42.4 \pm 14.7 \mathrm{c}$ \\
\hline
\end{tabular}

Means in the columns followed by the same letters do not differ significantly according to Duncan's multiple range test at $p=0.05$; the assessment of significance of differences was done for each genotype separately; means \pm SD 
Table 3. The effect of microcutting type and soil substrate type on ex vitro rooting and acclimatization of Lonicera caerulea 'Zojka' and 'Wojtek' in the greenhouse

\begin{tabular}{|c|c|c|c|c|c|c|c|}
\hline Cutting type & Soil substrate & $\begin{array}{c}\text { Rooted/survived } \\
\text { plants (\%) }\end{array}$ & $\begin{array}{l}\text { Fresh mass } \\
\text { of shoots } \\
\text { (mg) }\end{array}$ & $\begin{array}{l}\text { Shoot length } \\
\text { (mm) }\end{array}$ & $\begin{array}{c}\text { Fresh root } \\
\text { mass } \\
(\mathrm{mg})\end{array}$ & $\begin{array}{c}\text { Root } \\
\text { number/cutting }\end{array}$ & $\begin{array}{l}\text { Root length } \\
\text { (mm) }\end{array}$ \\
\hline \multicolumn{8}{|c|}{ 'Zojka' } \\
\hline \multirow{3}{*}{$\begin{array}{l}\text { Individual } \\
\text { shoots }\end{array}$} & peat & $88 \mathrm{a}$ & $91.9 \pm 20.0 \mathrm{c}$ & $81.4 \pm 7.0 \mathrm{a}$ & $22.0 \pm 8.7 \mathrm{c}$ & $2.8 \pm 1.1 \mathrm{a}$ & $25.6 \pm 5.6 \mathrm{a}$ \\
\hline & peat + sand & $80 \mathrm{a}$ & $52.9 \pm 9.0 \mathrm{~d}$ & $62.3 \pm 8.0 \mathrm{c}$ & $11.0 \pm 3.6 \mathrm{~d}$ & $2.7 \pm 0.7 \mathrm{a}$ & $24.4 \pm 5.8 \mathrm{a}$ \\
\hline & peat + perlite & $84 \mathrm{a}$ & $62.0 \pm 17.0 \mathrm{~cd}$ & $69.0 \pm 14.0 \mathrm{bc}$ & $15.4 \pm 8.3 \mathrm{~cd}$ & $2.5 \pm 0.7 \mathrm{a}$ & $23.7 \pm 7.1 \mathrm{a}$ \\
\hline \multirow{3}{*}{$\begin{array}{l}\text { Multiplied } \\
\text { clumps }\end{array}$} & peat & $68 \mathrm{a}$ & $127.7 \pm 41.0 \mathrm{~b}$ & $68.4 \pm 14.0 \mathrm{bc}$ & $22.8 \pm 8.7 \mathrm{c}$ & $1.8 \pm 0.5 b$ & $12.0 \pm 1.9 \mathrm{~b}$ \\
\hline & peat + sand & $72 \mathrm{a}$ & $139.3 \pm 75.0 \mathrm{ab}$ & $50.3 \pm 12.0 \mathrm{~d}$ & $33.0 \pm 21.3 b$ & $2.9 \pm 1.5 \mathrm{a}$ & $15.3 \pm 6.5 b$ \\
\hline & peat + perlite & $84 \mathrm{a}$ & $164.2 \pm 88.0 \mathrm{a}$ & $73.6 \pm 22.9 \mathrm{ab}$ & $49.6 \pm 24.4 \mathrm{a}$ & $3.0 \pm 0.7 \mathrm{a}$ & $21.8 \pm 7.5 \mathrm{a}$ \\
\hline \multicolumn{8}{|c|}{ 'Wojtek' } \\
\hline \multirow{3}{*}{$\begin{array}{l}\text { Individual } \\
\text { shoots }\end{array}$} & peat & 96 a & $97.8 \pm 20.0 b c$ & $106.8 \pm 14.9 \mathrm{a}$ & $20.9 \pm 6.6 c$ & $2.4 \pm 0.7 b$ & $27.1 \pm 7.4 \mathrm{a}$ \\
\hline & peat + sand & $80 \mathrm{ab}$ & $72.4 \pm 20.0 \mathrm{c}$ & $85.8 \pm 15.0 \mathrm{~b}$ & $12.9 \pm 6.9 \mathrm{c}$ & $1.9 \pm 0.4 \mathrm{c}$ & $29.4 \pm 8.1 \mathrm{a}$ \\
\hline & peat + perlite & $88 \mathrm{a}$ & $114.2 \pm 54.0 \mathrm{bc}$ & $112.3 \pm 29.0 \mathrm{a}$ & $24.0 \pm 9.5 b c$ & $1.9 \pm 0.5 \mathrm{c}$ & $21.8 \pm 4.5 b$ \\
\hline \multirow{3}{*}{$\begin{array}{l}\text { Multiplied } \\
\text { clumps }\end{array}$} & peat & $60 \mathrm{~b}$ & $133.9 \pm 34.0 \mathrm{~b}$ & $66.6 \pm 14.0 \mathrm{~cd}$ & $36.3 \pm 19.6 b$ & $2.0 \pm 0.5 b c$ & $22.0 \pm 6.8 b$ \\
\hline & peat + sand & $76 \mathrm{ab}$ & $103.4 \pm 18.0 \mathrm{bc}$ & $60.4 \pm 11.0 \mathrm{~d}$ & $20.0 \pm 7.1 \mathrm{c}$ & $1.6 \pm 0.7 \mathrm{c}$ & $10.8 \pm 2.9 \mathrm{c}$ \\
\hline & peat + perlite & $88 \mathrm{a}$ & $191.2 \pm 43.0 \mathrm{a}$ & $73.8 \pm 30.0 \mathrm{bc}$ & $71.8 \pm 33.0 \mathrm{a}$ & $3.1 \pm 1.0 \mathrm{a}$ & $28.4 \pm 12.1 \mathrm{a}$ \\
\hline
\end{tabular}

Note: See Table 2

Table 4. The genetic evaluation of acclimatized plantlets of Lonicera caerulea 'Wojtek' and 'Zojka' compared to mother plants

\begin{tabular}{|c|c|c|c|c|c|c|c|}
\hline \multirow[t]{2}{*}{ No. } & \multirow[t]{2}{*}{ Primer } & $\begin{array}{c}\text { Total } \\
\text { number } \\
\text { of bands }\end{array}$ & $\begin{array}{c}\text { Number } \\
\text { of polymorphic } \\
\text { bands }\end{array}$ & $\begin{array}{c}\text { Range } \\
\text { of amplicon } \\
(\mathrm{pb})\end{array}$ & $\begin{array}{l}\text { Total } \\
\text { number } \\
\text { of bands }\end{array}$ & $\begin{array}{c}\text { Number } \\
\text { of polymorphic } \\
\text { bands }\end{array}$ & $\begin{array}{c}\text { Range } \\
\text { of amplicon } \\
\text { (pb) }\end{array}$ \\
\hline & & \multicolumn{3}{|c|}{ 'Zojka' } & \multicolumn{3}{|c|}{ 'Wojtek' } \\
\hline 1 & UBC 825 & 9 & 1 & $500-1000$ & 11 & 0 & $400-1000$ \\
\hline 2 & UBC 810 & 6 & 0 & $600-1300$ & 5 & 0 & $500-1300$ \\
\hline 3 & UBC 827 & 12 & 0 & $300-800$ & 11 & 0 & $300-750$ \\
\hline 4 & UBC 865 & 9 & 0 & $300-1400$ & 13 & 0 & $300-1400$ \\
\hline Total & & 36 & 1 & & 40 & 0 & \\
\hline \multicolumn{2}{|c|}{ Proportion of polymorphic bands (\%) } & 2,77 & \multicolumn{5}{|c|}{0,00} \\
\hline 1 & Pst-CC/Mse-CC & 19 & 1 & $80-300$ & 16 & 1 & $50-300$ \\
\hline 2 & Pst-TT/Mse-CC & 35 & 1 & $250-900$ & 30 & 0 & $250-900$ \\
\hline 3 & Pst-GC/Mse-TA & 18 & 0 & $300-850$ & 16 & 0 & $400-850$ \\
\hline 4 & Pst-CG/Mse-AG & 42 & 0 & $200-760$ & 40 & 0 & $200-750$ \\
\hline Total & & 105 & 2 & & 102 & 1 & \\
\hline \multicolumn{2}{|c|}{ Proportion of polymorphic bands (\%) } & 1,90 & & & 0,98 & & \\
\hline
\end{tabular}


A

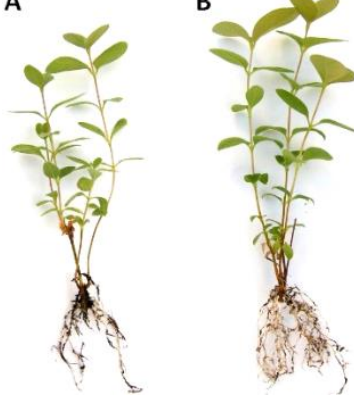

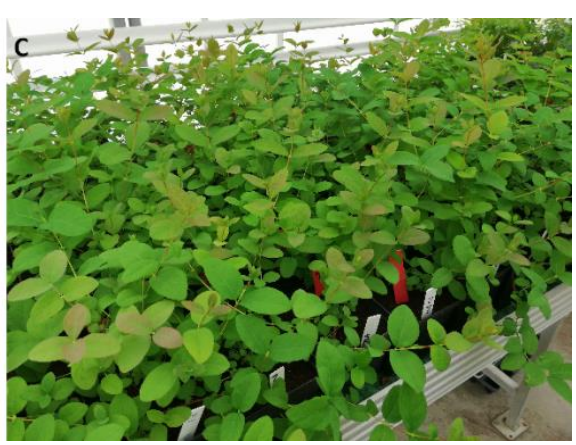

Figure 1. Plantlets of blue honeysuckle growing in the greenhouse: A - ex vitro rooted shoots of 'Wojtek' after 6 weeks of growth on the peat; $\mathrm{B}$ - ex vitro rooted shoots of 'Wojtek' after 6 weeks of growth on the peat and perlite; $\mathrm{C}$ - plants of 'Wojtek' and 'Zojka' after 2 years of growth ex vitro

\section{DISCUSSION}

Currently, in many commercial laboratories, microcuttings of several plant species are rooted directly, and root formation proceeds simultaneously with plantlet acclimatization. The costs of in vitro rooting, according to plant species, are calculated at $35-50 \%$ of the total micropropagation costs. Ex vitro rooting shortens the micropropagation time and reduces costs. So far, only the in vitro method has been successfully used to root blue honeysuckle shoots. During in vitro rooting, auxins were essential for achieving a high rooting percentage (Karhu 1997b; Sedlák \& Paprštein 2007; Dziedzic 2008; Krupa-Małkiewicz et al. 2017). On the other hand, auxin presence in the medium stimulated unwanted callus formation at the base of the rooted shoots (Karhu 1997b; Wojtania et al. 2018). We are in agreement with Karhu (1997b), who observed that abundant callus blocked the formation of new roots and decreased the survival rate of blue honeysuckle transferred to ex vitro conditions. A high rooting percentage and a good ex vitro survival and root growth of $L$. caerulea f. edulis microplants were achieved by a 7-day pulse treatment with $0.8 \mathrm{mg} \cdot \mathrm{dm}^{-3}$ IBA followed by rooting ex vitro (Karhu 1997b). Our study showed the possibility of successful ex vitro rooting of two L. caerulea var. kamtschatica cultivars 'Wojtek' and 'Zojka' without auxin treatments. It is worth noting that only $4 \%$ of 'Wojtek' and $40 \%$ of 'Zojka' microshoots were rooted in vitro if no auxin was supplied (Wojtania et al. 2018).
Higher ex vitro rooting frequency in soil substrate without auxin treatment as compared to the agar medium might be due to a specific greenhouse environment. Similarly, as in our study, peat alone or a mixture of peat and perlite/vermiculite was proved to be a better substrate for rooting and ex vitro growth of blue honeysuckle than peat and sand (Karhu 1997b; Dziedzic 2008; Krupa-Małkiewicz et al. 2017).

The success of micropropagation is clearly dependent on maintaining the genetic stability of propagules. The risk of genetic instability may be minimalized through plant production by axillary branching. Blue honeysuckle easily produced in vitro primary and secondary branches in response to cytokinin treatment. However, increased cytokinin concentration enhanced the growth of callus at the base of the explants and the spontaneous formation of adventitious shoots (Karhu 1997a). We observed no morphological differences between micropropagated and mother plants of blue honeysuckle 'Wojtek' and 'Zojka'. It is known that visible morphological variation occurs at a much lower frequency than at the DNA level (Evans \& Bravo 1986; Krishna et al. 2016). So, it is important to ensure that the micropropagation protocol does not brings changes at the molecular level (Cloutier \& Landry 1994; Krishna et al. 2016; Olhoft \& Phillips 1999). In the literature, there is no information on genetic stability of blue honeysuckle micropropagated plants. Available reports focused on genetic variation between species and cultivars belonging to genus Lonicera (Lamoureux et al. 2011; Naugžemys et al. 2011; Gawroński et al. 2014; He et al. 2016; Holubec et al. 2018). 
To our knowledge, this is the first assessment of DNA sequence variation in Polish cultivars of L. caerulea var. kamtschatica. Two PCR-based techniques have been used to test clonal stability because of their simplicity, cost-effectiveness, being highly informative and reliable (Arnau et al. 2002). The use of the two different molecular markers, which amplify different regions of the genome, gives more chances for the identification of genetic variations in the micropropagated clones (Martins et al. 2005). The number of bands generated was greater in AFLP than ISSR analysis. After 2 years of in vitro propagation, no polymorphism was detected for plantlets of 'Wojtek', in contrast to AFLP markers ( $0.98 \%$ of polymorphic bands). For 'Zojka' plants, the degree of variation was on average $2.8 \%$ and was comparable for AFLP and ISSR markers. Differences in the results obtained with the ISSR and AFLP markers probably reflect the different genomic regions amplified by the two marker types. Similar results were obtained by some other authors (Martins et al. 2005; Lakshmanan et al. 2007; Kour et al. 2014) who used molecular markers to confirm the genetic stability of micropropagated plantlets. On the basis of our results, it is not possible to clearly indicate which type of molecular marker used in this study is better for assessing genetic stability in the studied cultivars. We suggest using the two different types of markers, which amplify different regions of the genome, to increase the probability of detecting genetic variations in the micropropagated clones. In our research, combining the ISSR and AFLP data sets allowed a more comprehensive analysis of genetic stability of two Polish cultivars of blue honeysuckle and provided greater information about the genetic identity of micropropagated plants.

\section{Acknowledgments}

This study was conducted under the multiannual program "Actions to improve the competitiveness and innovation in the horticultural sector with regard to quality and food safety and environmental protection" of the Ministry of Agriculture and Rural Development (2015-2020).

\section{REFERENCES}

Arnau G., Lallemand J., Bourgoin M. 2002. Fast and reliable strawberry cultivar identification using inter simple sequence repeat (ISSR) amplification. Euphytica 129: 67-79. DOI: 10.1023/a:1021509206584.

Bednarek P.T., Orłowska R. 2020. Plant tissue culture environment as a switch-key of (epi)genetic changes. Plant Cell, Tissue and Organ Culture 140: 245-257. DOI: 10.1007/s11240-019-01724-1.

Borsai O., Hârța M., Szabo K., Kelemen C.D., Andrecan F.A., Codrea M.M., Clapa D. 2020. Evaluation of genetic fidelity of in vitro-propagated blackberry plants using RAPD and SRAP molecular markers. Horticultural Science 47: 21-27. DOI: 10.17221/20/2019-hortsci.

Cloutier S., Landry B.S. 1994. Molecular markers applied to plant tissue culture. In Vitro Cellular and Developmental Biology - Plant 30(1): 32-39. DOI: 10.1007/bf02632117.

Debnath S.C. 2011. Adventitious shoot regeneration in a bioreactor system and EST-PCR based clonal fidelity in lowbush blueberry (Vaccinium angustifolium Ait.). Scientia Horticulturae 128: 124-130. DOI: 10.1016/j.scienta.2011.01.012.

Debnath S.C. 2013. Propagation strategies and genetic fidelity in strawberries. International Journal of Fruit Science 13: 3-18. DOI: 10.1080/15538362.2012.696520.

Dziedzic E. 2008. Propagation of blue honeysuckle ( $L o$ nicera caerulea var. kamtschatica Pojark.) in in vitro culture. Journal of Fruit and Ornamental Plant Research 16: 93-100.

Evans D.A., Bravo J.E. 1986. Phenotypic and genotypic stability of tissue cultured plants. In: Zimmerman R.H., Griesbach R.J., Hammerschlag F.A., Lawson R.H. (Eds.), Tissue culture as a plant production system for horticultural crops. Current Plant Science and Biotechnology in Agriculture 2: 73-94. DOI: 10.1007/978-94-009-4444-2_6.

Gabryszewska E., Góraj-Koniarska J., Orlikowska T., Malinowski T., Markiewicz M., Wojtania A. 2016. Metodyka inicjacji i stabilizacji kultur oraz namnażania pędów jagody kamczackiej (Lonicera caerulea L. var. kamtschatica Sevast.) in vitro. Research Institute of Horticulture, Skierniewice, Poland, 13 p. [in Polish] 
Gawroński J., Hortyński J., Kaczmarska E., DyduchSiemińska M., Marecki W., Witorożec A. 2014. Evaluation of phenotypic and genotypic diversity of some Polish and Russian blue honeysuckle (Lonicera caerulea L.) cultivars and clones. Acta Scientiarum Polonorum, Hortorum Cultus 13(4): 157-169.

He H.Y., Zhang D., Qing H., Yang Y. 2016. Analysis of the genetic diversity of Lonicera japonica Thumb. using inter-simple sequence repeat markers. Genetics and Molecular Research 16(1); gmr16019338; 7 p. DOI: $10.4238 /$ gmr16019338.

Holubec V., Smekalova T., Leisova-Svobodova L. 2018. Morphological and molecular evaluation of the Far East fruit genetic resources of Lonicera caerulea L. - vegetation, ethnobotany, use and conservation. Genetic Resources and Crop Evolution 66(2): $121-$ 141. DOI: $10.1007 / \mathrm{s} 10722-018-0701-y$.

Hui J.X, Wen S.C., Hua Z.Y., Ming L.X. 2012. Comparative study on different methods for Lonicera japonica Thunb. micropropagation and acclimatization. Journal of Medicinal Plants Research 6(27): 4389-4393. DOI: 10.5897/jmpr011.1715.

Jain S.M. 2001. Tissue culture-derived variation in crop improvement. Euphytica 118: 153-166. DOI: 10.1023/a:1004124519479.

Jurikova T., Rop O., Mlcek J., Sochor J., Balla S., Szekeres L. et al. 2011. Phenolic profile of edible honeysuckle berries (genus Lonicera) and their biological effects. Molecules 17(1): 61-79. DOI: 10.3390/molecules17010061.

Karhu S.T. 1997a. Axillary shoot proliferation of blue honeysuckle. Plant Cell, Tissue and Organ Culture 48: 195-201. DOI: 10.1023/a:1005842022064.

Karhu S.T. 1997b. Rooting of blue honeysuckle microshoots. Plant Cell, Tissue and Organ Culture 48: 153-159. DOI: 10.1023/a:1005768117246.

Kour B., Kour G., Kaul S., Dhar M.K. 2014. In vitro mass multiplication and assessment of genetic stability of in vitro raised Artemisia absinthium L. plants using ISSR and SSAP molecular markers. Advances in Botany 2014; 727020; 7 p. $\quad$ DOI: 10.1155/2014/727020.

Krishna H., Alizadeh M., Singh D., Singh U., Chauhan N., Eftekhari M., Sadh R.K. 2016. Somaclonal variations and their applications in horticultural crops improvement. 3 Biotech 6; 54; 18 p. DOI: 10.1007/s13205-016-0389-7.
Krupa-Małkiewicz M., Ochmian I. 2014. Propagation of blue honeysuckles (Lonicera caerulea L.) in in vitro culture. Journal of Basic and Applied Sciences 10: 164-169. DOI: 10.6000/1927-5129.2014.10.22.

Krupa-Małkiewicz M., Ochmian I., Smolik M., Ostrowska K.M. 2017. Comparison of propagation method in in vitro and in vivo condition of Lonicera caerulea L. Folia Pomeranae Universitatis Technologiae Stetinensis 334(42)2: 79-88. DOI: 10.21005/aapz2017.42.2.09.

Lakshmanan V., Venkataramareddy S.R., Neelwarne B. 2007. Molecular analysis of genetic stability in long-term micropropagated shoots of banana using RAPD and ISSR markers. Electronic Journal of Biotechnology 10(1): 106-113. DOI: 10.2225/vol10issue1-fulltext-12.

Lamoureux D., Sorokin A., Lefèvre I., Alexanian S., Eyzaguirre P., Hausman J.-F. 2011. Investigation of genetic diversity in Russian collections of raspberry and blue honeysuckle. Plant Genetic Resources $\quad 9(2)$ : 202-205. DOI: $10.1017 / \mathrm{s} 1479262111000323$.

Martins M., Sarmento D., Oliveira M. 2005. Genetic stability of micropropagated almond plantlets, as assessed by RAPD and ISSR markers. Plant Cell Reports 23: 492-496. DOI: 10.1007/s00299-0040870-3.

Murashige T., Skoog F. 1962. A revised medium for rapid growth and bio assays with tobacco tissue cultures. Physiologia Plantarum 15: 473-497. DOI: 10.1111/j.1399-3054.1962.tb08052.x.

Naugžemys D., Žilinskaitė S., Kleizaitė V., Skridaila A., Žvingila D. 2011. Assessment of genetic variation among elite and wild germplasm of blue honeysuckle (Lonicera caerulea L.). Baltic Forestry 17: $8-16$.

Olhoft P.M., Phillips R.L. 1999. Genetic and epigenetic instability in tissue culture and regenerated progenies. In: Lerner H.R. (Ed.), Plant Responses to Environmental Stresses. Books in soils, plants, and the environment 72: 111-148. DOI: 10.1201/9780203743157-7.

Plekhanova M.N. 2000. Blue honeysuckle (Lonicera caerulea L.) - a new commercial berry crop for temperate climate: Genetic resources and breeding. Acta Horticulturae 538: 159-164. DOI: 10.17660/actahortic.2000.538.25. 
Podwyszyńska M., Niedoba K., Korbin M., Marasek A. 2006. Somaclonal variation in micropropagated tulips determined by phenotype and DNA markers. Acta Horticulturae 714: 211-220. DOI: 10.17660/actahortic.2006.714.23.

Poyarkova A.I. 2000. Lonicera L. In: Schischkin B.K. (Ed.), Flora of the USSR, vol. 23. Science Publishers, Moscow, Russia, pp. 446-549.

Sedlák J., Paprštein F. 2007. In vitro propagation of blue honeysuckle. Horticultural Science 34(4): 129-131. DOI: 10.17221/1871-hortsci.

Smulders M.J.M., de Klerk G.J. 2011. Epigenetics in plant tissue culture. Plant Growth Regulation 63: 137-146. DOI: 10.1007/s10725-010-9531-4.
Svarcova I., Heinrich J., Valentova K. 2007. Berry fruits as a source of biologically active compounds: The case of Lonicera caerulea. Biomedical Papers 151(2): 163-174. DOI: 10.5507/bp.2007.031.

Vos P., Hogers R., Bleeker M., Reijans M., van de Lee T., Hornes M. et al. 1995. AFLP: a new technique for DNA fingerprinting. Nucleic Acids Research 23: 4407-4414. DOI: 10.1093/nar/23.21.4407.

Wojtania A., Matysiak B., Góraj-Koniarska J., Kiszczak W., Kucharska D., Kowalska U. 2018. Ukorzenianie i aklimatyzacja mikrosadzonek truskawki, maliny, jagody kamczackiej i czosnku w warunkach ex vitro - raport z badań wykonanych w 2018 roku. Research Institute of Horticulture, Skierniewice, Poland, 16 p. [in Polish] 